\title{
An Exploratory Evaluation of UAS Detect and Avoid Operations in the Terminal Environment
}

\author{
Lisa Fern ${ }^{1}$, and R. Conrad Rorie ${ }^{2}$ \\ NASA Ames Research Center, Moffett Field, CA, 94035 \\ Zachary Roberts ${ }^{3}$ \\ San Jose State University, Moffett Field, CA, 94035 \\ and \\ Kevin J. Monk ${ }^{4}$ \\ NASA Ames Research Center, Moffett Field, CA, 94035
}

\begin{abstract}
New technical standards for Unmanned Aircraft Systems (UAS) detect and avoid (DAA) systems mark recent progress toward realizing the goal of full integration of UAS into the National Airspace System (NAS). The DAA system is intended to provide a means of compliance with operating regulations that required pilots on board manned aircraft to remain "well clear" of other aircraft which is accomplished through out-the-window visual acquisition of other aircraft and application of a subjective judgment of safe separation. The requirements for the DAA system, including the specification of a DAA well clear threshold as well as functional requirements for detecting, tracking, alerting and guidance processing, and displays, are specified in DO-365, Minimum Operational Performance Standards (MOPS) for DAA Systems developed within RTCA Special Committee 228 (SC-228). Intended as the first in a series of phased versions, these requirements are frequently referred to as the "Phase 1" DAA system. The Phase 1 DAA system is limited for use by aircraft transitioning to and from Class $A$ or special use airspace, through Class D, E, and G airspace. In particular, the Phase 1 DAA MOPS are not intended for terminal airspace operations, a critical gap for enabling a full range of UAS operations. The application of the Phase 1 DAA system and DAA well clear threshold within the terminal area is predicted to result in a high number of unnecessary alerts when the UAS is safely separated from other traffic. The goal of the present study was to examine pilot performance and operational issues related to the operation of the Phase 1 DAA system in a terminal area. This experiment was intended as an exploratory study that would be used to inform the development of a new terminal area-specific DAA well clear definition, and associated alerting and guidance requirements. The two main objectives of this study were to: 1) characterize pilot behavior in the terminal environment with the Phase 1 DAA system, and 2) investigate the effect of modifications to the Phase 1 DAA alerting and guidance structure. In particular, the authors were interested in determining whether the removal of specific alerting and guidance levels, without changing the DAA well clear definition or alerting thresholds, would impact pilot performance while conducting terminal operations. The results indicate that the Phase 1 well clear definition and alerting and guidance resulted in frequent alerting that degraded pilots' ability to discriminate between encounters where another aircraft was safely separated versus when a maneuver was necessary. The resulting impact on pilot performance was slower response times and higher frequency and severity of losses of DAA well clear compared to those observed for experiments examining pilot performance in the en route environment. There was no significant effect of alerting and guidance display configuration on pilot performance.
\end{abstract}

\footnotetext{
${ }^{1}$ Aerospace Engineer, Aviation Systems Division, MS 262-2, AIAA Member.

${ }^{2}$ General Engineer, Aviation Systems Division, MS 262-2, AIAA Member.

${ }^{3}$ Research Associate, Human Systems Integration, MS 262-2.

${ }^{4}$ General Engineer, Human Systems Integration, MS 262-2.
} 


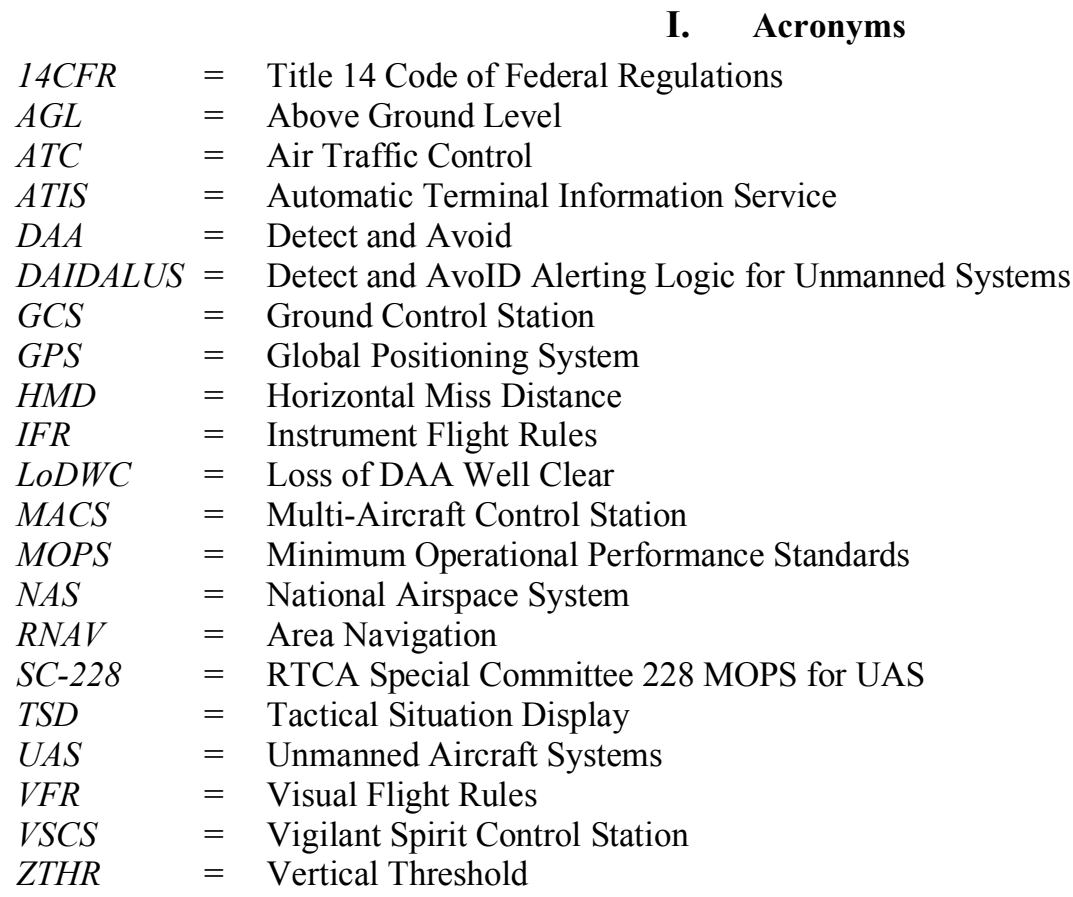

\section{Introduction}

Recent research and technology development efforts have resulted in significant progress toward realizing the goal of full integration of Unmanned Aircraft Systems (UAS) into the National Airspace System (NAS). This progress is most evident in recently published performance standards documents for critical technologies considered essential to enabling safe UAS operations $[1,2,3]$. One of these technologies, essential for ensuring safe integration with existing aircraft in the NAS, is the detect and avoid (DAA) system. The DAA system was developed to provide a means of compliance with the operating rules of Title 14 of the Code of Federal Regulations (14 CFR) which address see and avoid, collision avoidance, and right-of-way rules [4]. These operating regulations assume that a pilot onboard an aircraft is able to exercise her authority to fully comply with these rules by means of out-the-window visual acquisition of nearby aircraft and subjective judgment of safe separation, or "well clear". The UAS DAA system uses electronic detection through onboard surveillance equipment to track nearby aircraft and provide alerting and maneuver guidance information to the ground-based pilot to assist her in remaining "DAA well clear" - a mathematically derived separation criteria that will be used by UAS operating in the NAS5.

A key component of the DAA system is the provision of a multi-level alerting and maneuver guidance structure designed to assist the remote pilot, situated at a ground-based control station (GCS), in deciding when and how to execute an avoidance maneuver to remain DAA well clear of other aircraft. The DAA alerting and guidance thresholds are based on predicted time to penetration of the DAA well clear threshold (also referred to as a loss of DAA well clear), taking into account pilot response time, aircraft maneuver time, and various system latencies. The DAA alerting structure is designed to give the pilot temporal information about when a maneuver to remain DAA well clear is necessary. A DAA corrective alert is a caution (yellow) level alert that indicates to the pilot that there is sufficient time to contact air traffic control (ATC) prior to maneuvering to avoid a loss of DAA well clear; whereas a DAA warning alert is a warning (red) level alert that indicates to the pilot that they need to maneuver immediately to avoid a loss of DAA well clear. DAA maneuver guidance, on the other hand, is designed to provide information to the pilot on what maneuvers are safe for maintaining DAA well clear - corrective maneuver guidance indicates trajectories that will result in triggering a corrective alert (and therefore a predicted loss of DAA well clear with sufficient time to coordinate a maneuver with ATC), while warning maneuver guidance indicates vertical and horizontal trajectories that will result in a warning alert (and an imminent loss of DAA well clear). Green bands, or the absence of warning or corrective bands, indicate trajectories that are predicted to result in the ownship remaining DAA well clear within the prescribed look-ahead time. Thus, the DAA well clear threshold and alerting and maneuver guidance thresholds are tightly coupled. The requirements for the display of DAA alerting and guidance information, including the structure (i.e., levels), thresholds, and presentation format, were derived through a series 
of human-in-the-loop experiments that evaluated the effect of different DAA display configurations on pilot performance in remaining DAA well clear $[6,7,8,9,10]$. An example depiction of a DAA display is shown in Fig. 1.

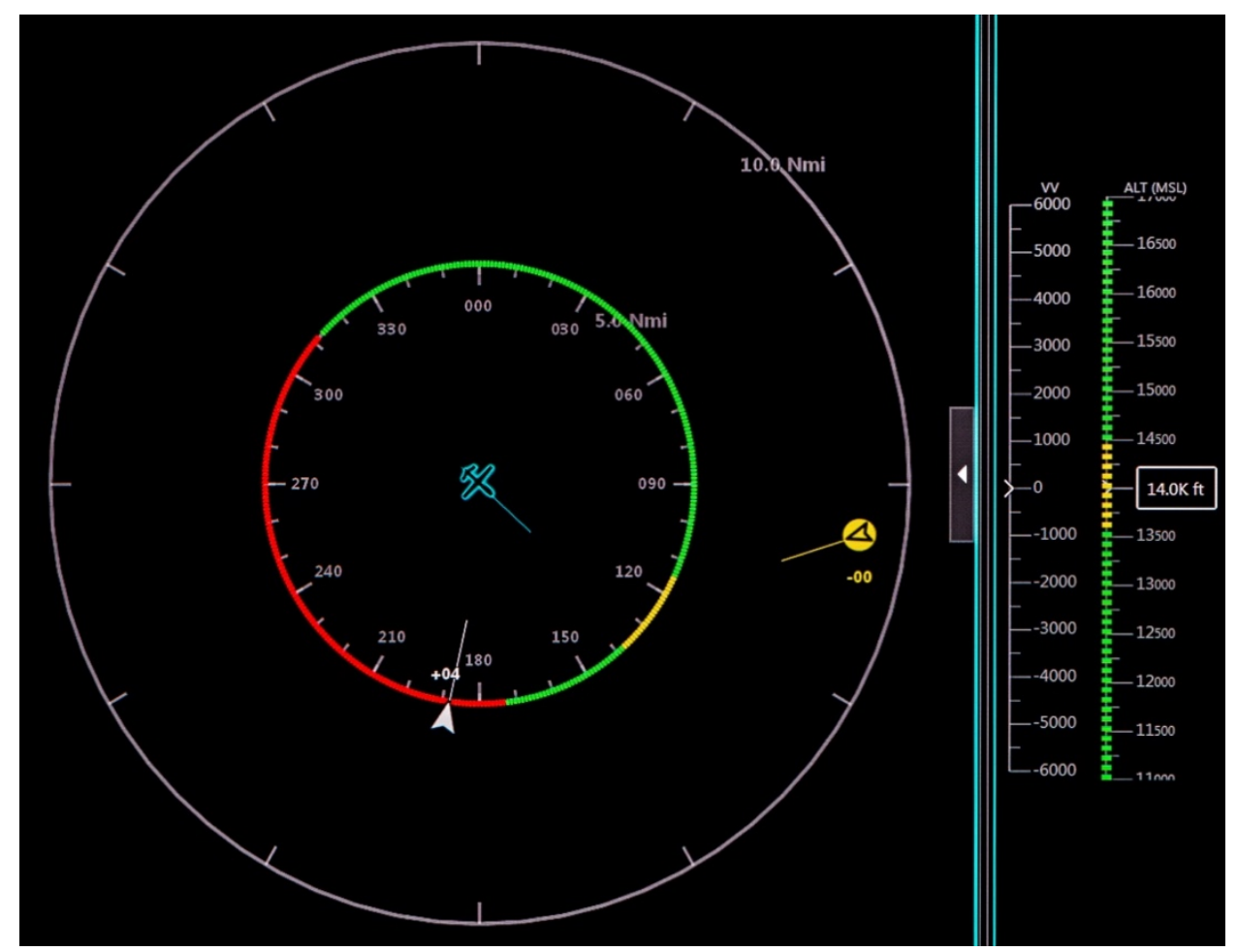

Fig. 1 An example depiction of the DAA alerting and maneuver guidance. In this example, a caution-level alert (yellow, circular symbol with a chevron inlay) is presented in conjunction with caution and warning level maneuver guidance, depicted as "bands", on the inner range ring (center) and altitude tape (right).

The requirements for the DAA system, including the specification of a DAA well clear threshold as well as functional requirements for detecting, tracking, alerting and guidance processing, and displays, are specified in DO365, Minimum Operational Performance Standards (MOPS) for DAA Systems developed within RTCA Special Committee 228 (SC-228) [2]. Intended as the first in a series of phased versions, these requirements are frequently referred to as the "Phase 1" DAA system. While the development of DAA standards is a key progress marker for UAS integration in the NAS, the Phase 1 DAA system is limited for use by aircraft transitioning to and from Class A or special use airspace [higher than 500' Above Ground Level (AGL)], through Class D, E, and G airspace. In particular, the DAA MOPS are not intended for terminal airspace operations, such as takeoff and landing or transiting through Class B and C airspace, a critical gap for enabling a full range of UAS operations. Part of SC228's "Phase 2" efforts will address, at a minimum, straight-in Instrument Flight Rules (IFR) takeoff and landing operations at towered and non-towered airfields in Class C, D, E and G airspace, including when other aircraft are in the Visual Flight Rules (VFR) traffic pattern [11].

It is expected that an essential part of the work to enable UAS terminal operations will include the development of a DAA well clear threshold specific to the terminal environment where aircraft are operating much closer together than in the en route environment. For this integration to be seamless and interoperable, it will be important to achieve a balance between ensuring safe separation between UAS and manned aircraft while also minimizing disruptions to the efficient operation of both manned and unmanned aircraft in terminal airspace.

The application of the Phase 1 DAA system and DAA well clear threshold within the terminal area is predicted to result in a high number of unnecessary alerts when the UAS is safely separated from other traffic. As depicted in Fig. 2, it is possible for some runway and VFR pattern configurations to be within the $4000 \mathrm{ft} / 0.66 \mathrm{NM}$ minimum horizontal spatial threshold of the DAA well clear definition. A UAS landing at such a runway with traffic in the VFR pattern could have an actual or predicted loss of DAA well clear, despite being considered safely separated given the standard procedures of that airfield. This level of spacing with the Phase 1 system would result in alerts and guidance on the DAA display that indicate to the pilot that a maneuver is necessary. Thus, pilots would be forced to either comply with the Phase 1 DAA system and maneuver against safely-separated traffic, or disregard 
the DAA alerting and guidance, which could unintentionally influence how they use the system in en route operations.

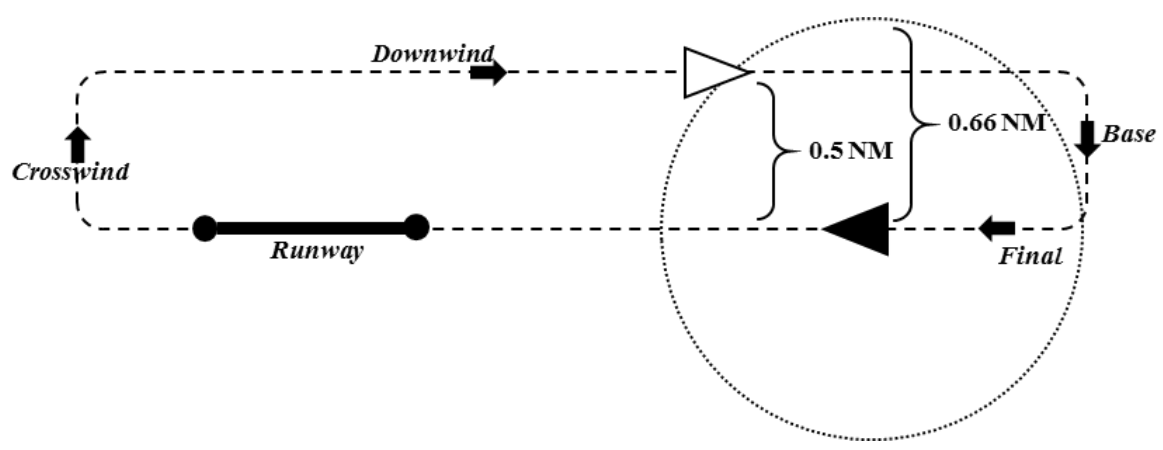

Fig. 2 Depiction of a hypothetical VFR traffic pattern, with VFR traffic (white triangle) on the downwind and

a UAS (black triangle) on final. As shown, the VFR intruder will generate DAA alerting and guidance because it is within the horizontal threshold of the Phase 1 DAA well clear definition (dashed circle).

The goal of the study presented in this paper was to examine pilot performance and operational issues related to the operation of the Phase 1 DAA system in a terminal area. This experiment was intended as an exploratory study that would be used to inform the development of a new terminal area-specific DAA well clear definition, and associated alerting and guidance requirements. The two main objectives of this study were to: 1) characterize pilot behavior in the terminal environment with the Phase 1 DAA system, and 2) investigate the effect of modifications to the Phase 1 DAA alerting and guidance structure. In particular, the authors were interested in determining whether the removal of specific alerting and guidance levels, without changing the DAA well clear definition or alerting thresholds, would impact pilot performance while conducting terminal operations.

\section{Method}

\section{A. Experimental Design}

This study employed a one-way between subject factorial experimental design to compare pilot performance with three levels (D1-D3) of DAA alerting and guidance displays based on the Phase 1 DAA requirements. As shown in Fig. 3, D3 consisted of the full Phase 1 DAA alerting, while D2 suppressed the presentation of corrective level alerting, and D1 suppressed the presentation of both the corrective and warning level alerting. Suppressed corrective and warning alerts were presented instead as preventive alerts.
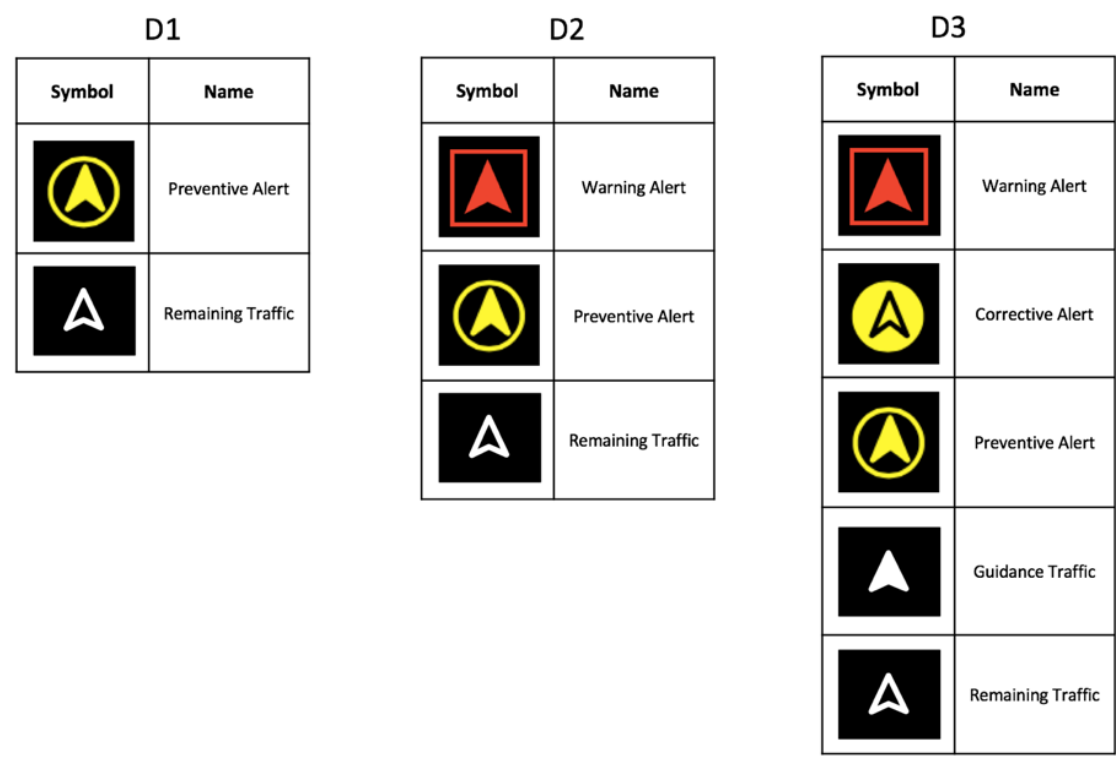

Fig. 3 The three DAA alerting structures used in each of the three display conditions, D1, D2 and D3. 
The maneuver guidance presentation was also modified to be consistent with the alerting structure in each condition. Per the Phase 1 MOPS, corrective and warning maneuver guidance information is provided along with corrective and warning alerts. Therefore, the corrective and warning alerts contained in conditions D2 and D3 were accompanied with corrective and warning maneuver guidance to remain DAA well clear, respectively. In addition, D3 also contained warning level maneuver guidance to regain DAA well clear. There was no maneuver guidance present in the D1 display condition. Fig. 4, Fig. 5, and Fig. 6 and show the progression of DAA alerts and guidance as an encounter progresses in time from no Loss of DAA well clear (LoDWC) predicted until a LoDWC is unavoidable for each of the three display conditions.

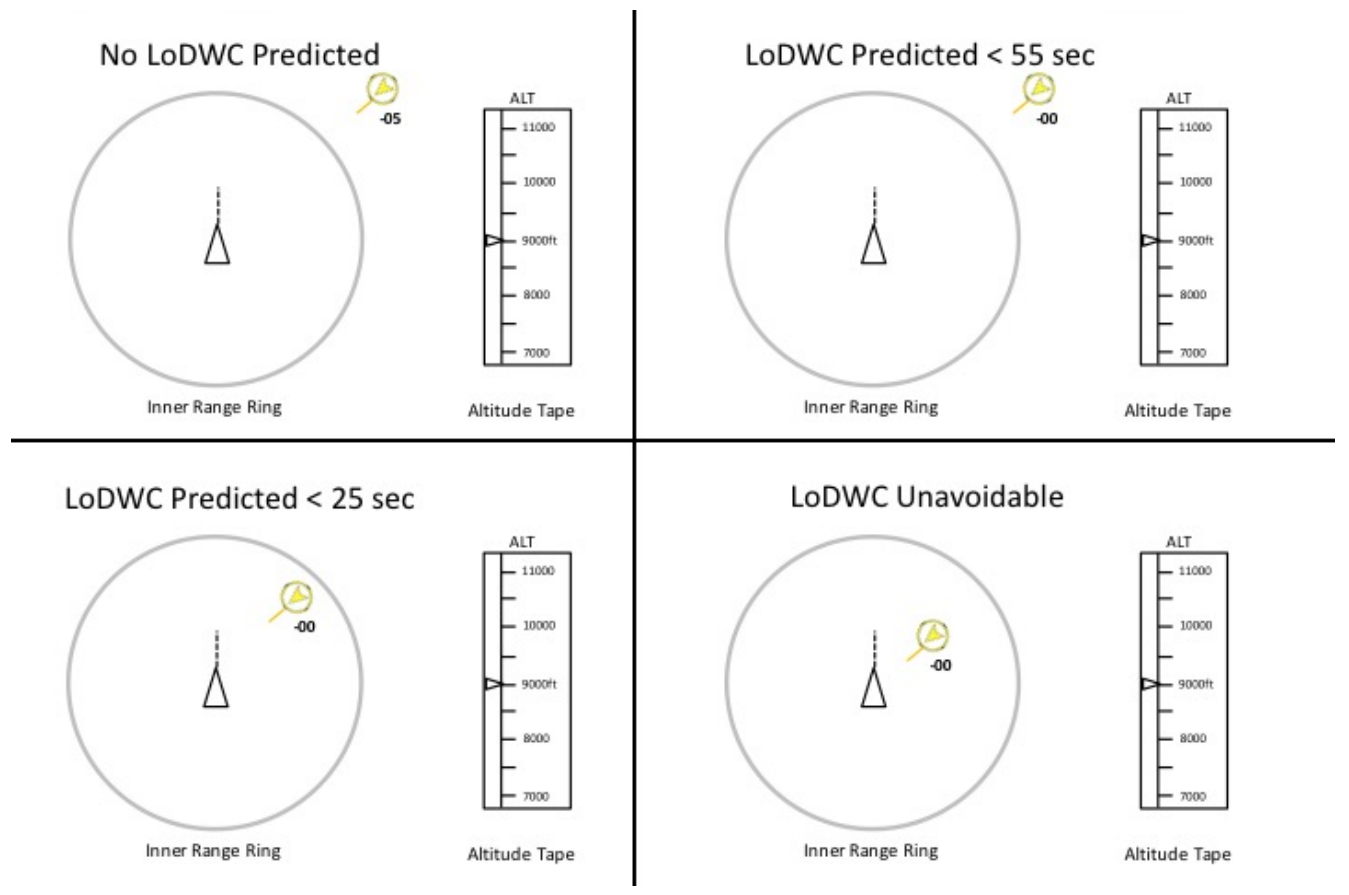

Fig. 4 Alert and guidance progression example, for the D1 display condition.

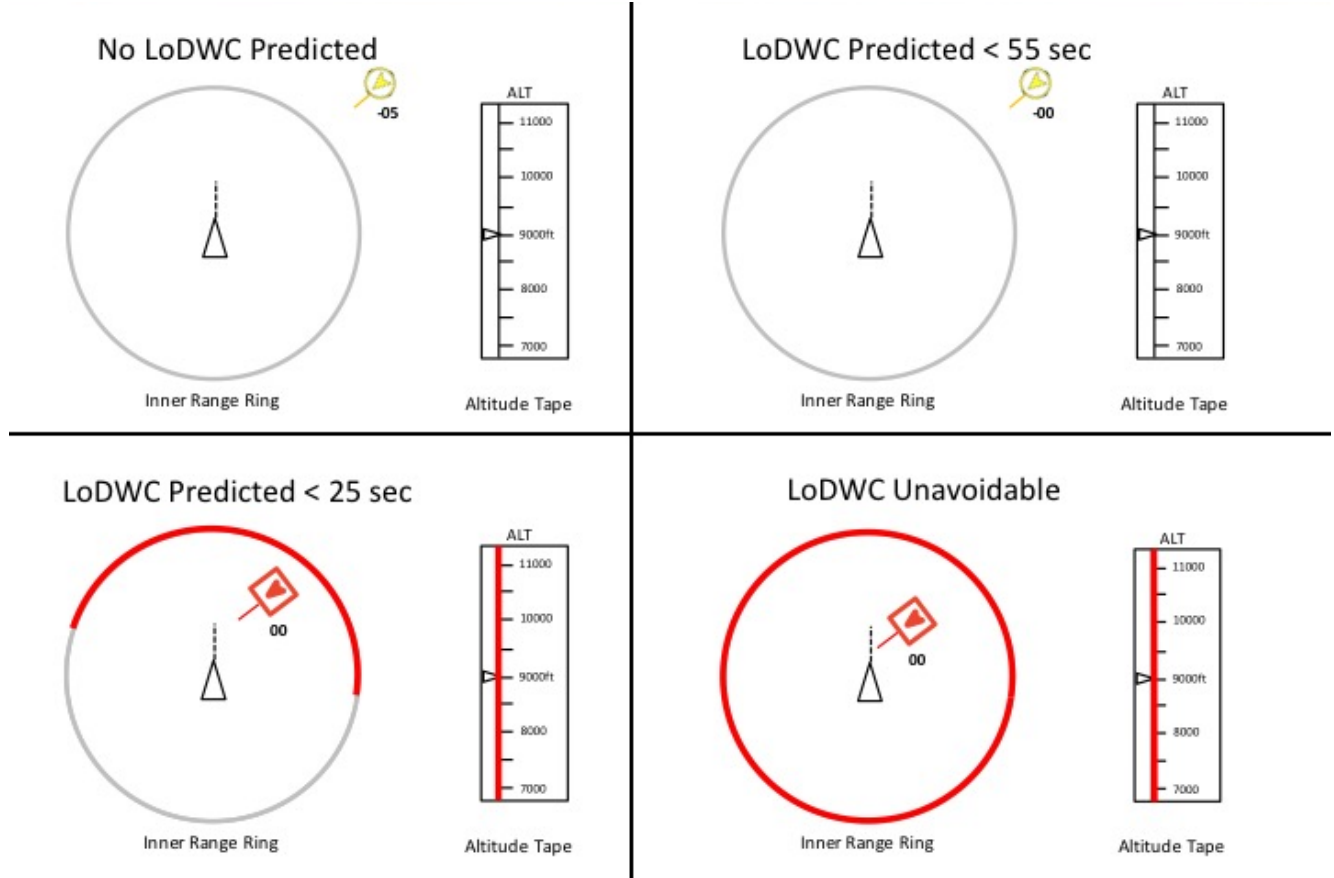

Fig. 5 Alert and guidance progression example, for the D2 display condition. 


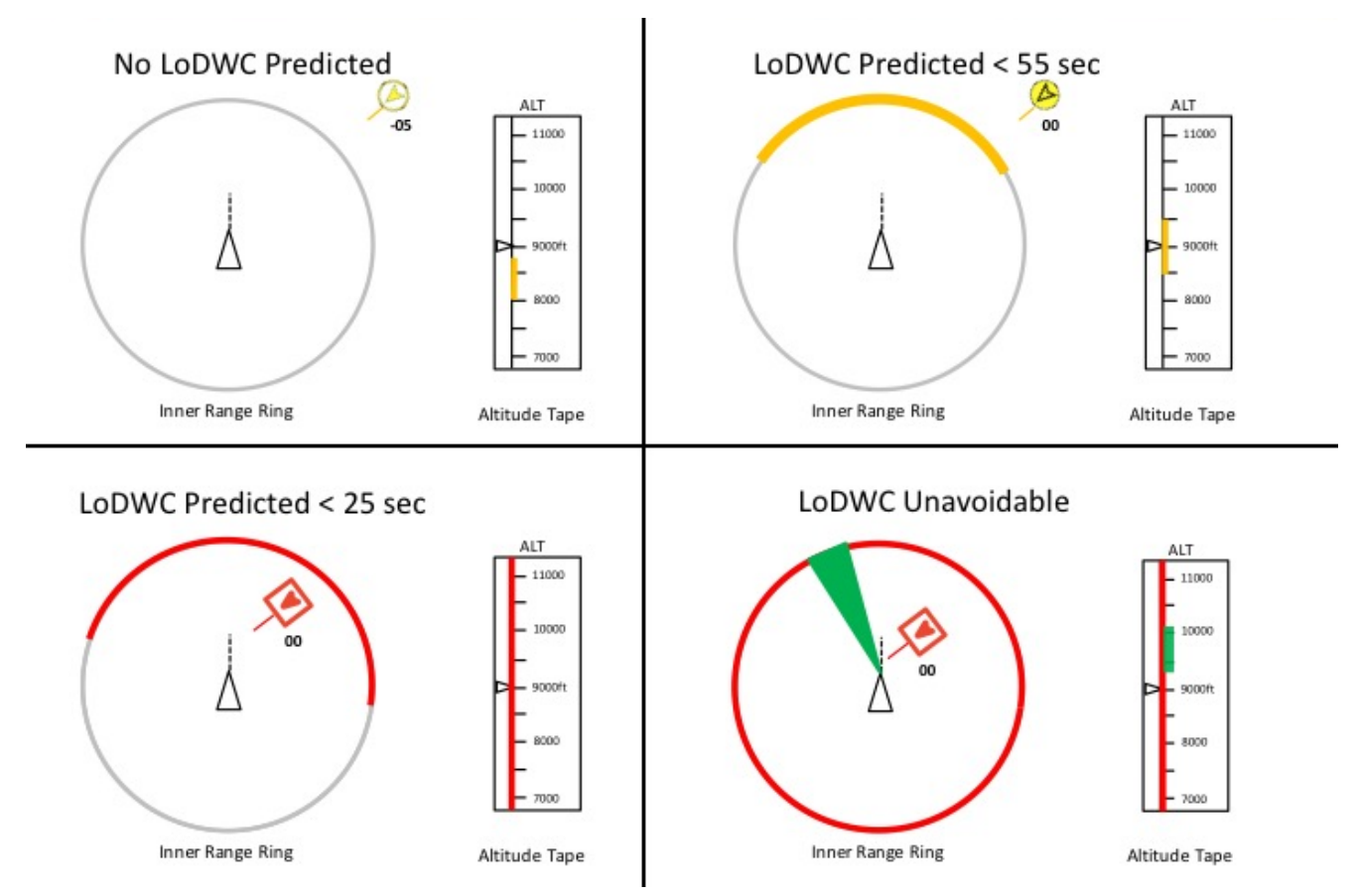

Fig. 6 Alert and guidance progression example, for the D3 display condition.

\section{B. Participants}

A total of 18 pilots $(M=39$ years of age) were recruited to participate in this study. All pilots had manned flight experience ( $M=2,200$ civilian flight hours) and were rated for IFR. Nine of the recruited pilots also had experience flying unmanned aircraft ( $M=1,100$ combined military combat and non-combat flight hours). Three different retired ATC served as simulation confederates. One ATC, with experience as a tower controller, served as the confederate tower controller for the duration of the experiment. The other two ATC, both with experience as center controllers, alternated as the confederate center controller over the course of the study. Four current general aviation pilots served as confederate "pseudo" pilots and controlled the simulated manned traffic in the study.

\section{Simulation Environment}

\section{Ground Control Station}

The GCS under test consisted of two primary components. The first was the Air Force Research Laboratory's Vigilant Spirit Control Station (VSCS), which was made up of four different displays: the tactical situation display (TSD), the DAA traffic display, the status panel, and the approach chart viewer [12]. The TSD served as pilots' primary display and contained the navigation information and vehicle control interfaces necessary for controlling the aircraft. The vehicle control interfaces supported two control modes - "Navigation Mode" (NAV) and "Holds Mode" (HOLDS). HOLDS mode enabled a heading, altitude and speed hold when engaged, and was primarily used to transition from center to terminal airspace and to make maneuvers for traffic avoidance. NAV mode was enabled to fly the final leg of the approach along the specified glide slope. The DAA display contained all DAA-related information and showed all traffic within the simulated sensor ranges as well as their given alert status and associated DAA guidance. All vehicle control inputs to the ownship, however, had to be made via the TSD. The status panel provided additional telemetry and system data, along with an electronic approach and landing checklist. Finally, the approach chart viewer showed an electronic version of the RNAV GPS approach plate into the experimental airport.

The second major GCS component was a touchscreen voice communications tablet, which allowed pilots to individually transmit, or receive, on the experimental center, tower, and Automatic Terminal Information Service (ATIS) frequencies. Pilots were connected to the tablet via a wireless, push-to-talk headset.

\section{Experimental Airspace}

The Multi-Aircraft Control Station (MACS) software suite was used to provide the experimental airspace and confederate displays. For the purposes of this study, MACS was configured to simultaneously support Oakland Center airspace (ZOA 40/41) and Santa Rosa airport (KSTS), with representative traffic flying in both regions. 
High-fidelity ATC radar displays were generated by MACS for the center and tower confederate controllers. MACS also generated four pseudo-pilot stations that allowed pilots to manage traffic in both Oakland center airspace and traffic flying in and around the VFR traffic pattern at KSTS.

Santa Rosa airport was configured within MACS to have a single active runway (runway 14/32). The tower controller and pseudo pilot displays included graphical references for the simulated VFR traffic pattern, the parameters of which were designed according to feedback from active KSTS tower controllers. The traffic pattern altitude was set to $1,150 \mathrm{ft}$ and had left and right downwind legs with different offsets relative to the runway. The right downwind leg had an approximate lateral offset of $0.5 \mathrm{NM}$ from the runway, while the left downwind leg had an approximate $1.5 \mathrm{NM}$ offset. The right downwind leg, therefore, was positioned inside of the DAA well clear horizontal threshold, meaning traffic could trigger the DAA system alerting and guidance if it was established on the right downwind while the UA was on a straight-in final approach.

Table 1. Full Phase 1 MOPS DAA system alerting structure.

\begin{tabular}{|c|c|c|c|c|c|}
\hline Alert Level & Separation Criteria & $\begin{array}{l}\text { Time to Loss of } \\
\text { DAA Well Clear }\end{array}$ & Icon & $\begin{array}{c}\text { Aural Alert } \\
\text { Verbiage }\end{array}$ & $\begin{array}{l}\text { Expected Pilot } \\
\text { Response }\end{array}$ \\
\hline $\begin{array}{l}\text { DAA Warning } \\
\text { Alert* }\end{array}$ & $\begin{array}{c}\mathrm{HMD}=0.66 \mathrm{~nm} \\
\mathrm{ZTHR}=450 \mathrm{ft} \\
\operatorname{modTau}=35 \mathrm{sec}\end{array}$ & $25 \mathrm{sec}$ & & $\begin{array}{c}\text { "Traffic, } \\
\text { Maneuver Now; } \\
\text { Traffic, } \\
\text { Maneuver Now" }\end{array}$ & $\begin{array}{l}\text { Maneuver } \\
\text { immediately }\end{array}$ \\
\hline $\begin{array}{c}\text { Corrective DAA } \\
\text { Alert** }\end{array}$ & $\begin{array}{l}\mathrm{HMD}=0.66 \mathrm{~nm} \\
Z \mathrm{THR}=450 \mathrm{ft} \\
\operatorname{modTau}=35 \mathrm{sec}\end{array}$ & $55 \mathrm{sec}$ & & "Traffic, Avoid" & $\begin{array}{c}\text { Maneuver } \\
\text { following ATC } \\
\text { approval }\end{array}$ \\
\hline $\begin{array}{c}\text { Preventive DAA } \\
\text { Alert }\end{array}$ & $\begin{array}{c}\mathrm{HMD}=0.66-1.0 \mathrm{~nm} \\
\mathrm{ZTHR}=450-700 \mathrm{ft} \\
\operatorname{modTau}=35 \mathrm{sec}\end{array}$ & N/A & & $\begin{array}{l}\text { "Traffic, } \\
\text { Monitor" }\end{array}$ & $\begin{array}{l}\text { Monitor traffic; } \\
\text { maneuver not } \\
\text { currently required }\end{array}$ \\
\hline $\begin{array}{c}\text { Guidance Traffic } \\
\text { Alert }\end{array}$ & $\begin{array}{l}\text { Associated with } \\
\text { banding outside } \\
\text { current course }\end{array}$ & $\mathrm{N} / \mathrm{A}$ & & $\mathrm{N} / \mathrm{A}$ & $\begin{array}{l}\text { No maneuver } \\
\text { required }\end{array}$ \\
\hline None (Target) & $\begin{array}{l}\text { Within surveillance } \\
\text { field of regard }\end{array}$ & $\mathrm{N} / \mathrm{A}$ & & N/A & $\begin{array}{l}\text { No maneuver } \\
\text { required }\end{array}$ \\
\hline
\end{tabular}

* The DAA Warning alert was only visible in display conditions D2 and D3.

** The Corrective DAA alert was only visible in display condition D3.

\section{Detect and Avoid System}

The Detect and Avoid Alerting Logic for Unmanned Systems (DAIDALUS) served as the DAA processor for the current study [13]. DAIDALUS, developed at NASA Langley Research Center, references configurable separation and alerting parameters to generate DAA alerts and guidance. For the purposes of the present experiment, DAIDALUS was configured to define a loss of DAA well clear according to the Phase 1 MOPS definition: 0.66NM horizontal miss distance (HMD) and distance modifier (DMOD), $\pm 450 \mathrm{ft}$ vertical threshold (ZTHR), and 35 second modified Tau (modTau). Alerts were determined based on constant state extrapolation for both ownship and intruders. The different types of DAA alerts and guidance are shown above in Figures 3-6. The specific parameters for the entire Phase 1 MOPS DAA alerting structure is shown in Table 1.

\section{Procedure}

\section{Training}

Pilot training consisted of three general phases. The first phase of training covered the experimental protocol (e.g., run schedule and a high-level explanation of their role in the experiment) and basic VSCS functionality. The essential VSCS functions pilots were trained on included how to control their UA in both the NAV and HOLDS modes, as well as how to transition safely between them. They were also trained on how to customize their GCS, such as the placement of the primary vehicle control interface, the type of map used as the background of the TSD (sectional chart vs. IFR low altitude chart), and the orientation of both the TSD and DAA display (north up vs. track up). The final portion of the first phase of training included how to use the approach and landing checklist, which allowed pilots to 'check off' the items they had completed, as well as how to manage their frequencies on the provided voice-comm panel. 
The second phase of training addressed the DAA system. Pilots received detailed, hands-on training covering each alert level and guidance type available in their condition. Pilots in the D1 condition, where the corrective and warning alerts were suppressed, were taught that preventive DAA alerts may or may not lead to a loss of DAA well clear. Pilots in the D2 condition, where only the corrective alert was suppressed, were taught that imminent losses of DAA well clear (i.e., within 25 seconds) would generate a warning alert. Lastly, pilots in the D3 condition were trained on the full Phase 1 MOPS alerting and guidance structure, which included both corrective and warning DAA alerts and guidance, as well as guidance to regain DAA well clear (which appeared only once a loss of DAA well clear could not be avoided). Pilots in all conditions were notified that the Phase 1 DAA well clear definition was designed for en route operations and may or may not perform appropriately in the terminal area. Pilots were trained to use their discretion at all times.

At the end of the second phase of the training, pilots completed a 20-minute practice scenario where they responded to a variety of encounters. Researchers verified at this time that pilots both fully understood the alert structure and knew how to control the aircraft to safely avoid conflicts. In the event that pilots were not yet sufficiently trained on VSCS or DAA functionality, they received a second practice scenario.

The third and final phase of training covered how to fly the type of approach the pilot was going to subsequently run in the experimental trial. Pilots flew three different approach types over the course of the simulation (described in detail below) and received the relevant training immediately before a given experimental run. Pilots were trained first on the general procedures for the approach, and then flew two practice approaches before concluding training.

2. Pilot Task and Scenarios

Pilots were tasked with utilizing the DAA system to safely fly three different types of approach into runway 14 at KSTS: Instrument Approach, Visual Approach, and VFR Traffic Pattern. All three approach types were conducted under IFR but required pilots to interact with the GCS in unique ways. In the instrument approach scenario, pilots performed an Area Navigation (RNAV) Global Positioning System (GPS) approach, which was largely accomplished through hands-off, waypoint-to-waypoint navigation that allowed the UAS to fly the published glide slope. In the visual approach scenario, pilots had to upload desired changes to the commanded heading, altitude and/or speed of the UAS to position itself for a $3 \mathrm{~nm}$ straight-in approach to the runway. In the VFR pattern scenario, pilots were required to send uploads to the aircraft in HOLDS mode to safely enter the right or left downwind of the VFR traffic pattern.

Pilots operated a simulated MQ-9 Reaper and gained control of the aircraft with at level altitude in Oakland Center airspace. Their first responsibility was to coordinate a hand-off from the Oakland center's frequency to the KSTS tower frequency. Once on the tower frequency, they had to request, and gain approval, for their desired approach. Pilots were simultaneously completing an approach and landing checklist, which required them to verify their runway, brief their descent profile, check the Automatic Terminal Information Service (ATIS), and lower their landing gear. Researchers were positioned next to the pilot participants in order to confirm that pilots successfully completed each step of the checklist.

Aside from the task of flying the approach into KSTS, pilots were expected to react to scripted conflicts, each intended to excite the DAA system. Half of the scripted conflicts were designed to occur before the UAS reached the final approach fix, and half were scripted to occur once the UAS was established on final. Pilots were responsible for determining whether or not the DAA system was alerting them to a legitimate threat, and whether it was necessary to abandon the approach. As has been explained, the DAA system under test was not designed to account for the closer spacing of the terminal area, so pilots relied entirely on their discretion. In addition to intruders that were scripted to directly interact with the UAS, there were also simulated manned aircraft that were flying into KSTS nominally, with no intention of coming into conflict with the UAS.

3. Experimental Trials

Each participant completed three 75-minute experimental trials using one of the three alerting and guidance display configurations (D1, D2, or D3). The trials were blocked, and counter-balanced, by approach type. Within a single trial, pilots flew six approaches, each lasting roughly 12 minutes. The approach started in Oakland center airspace and concluded when the UAS reached the runway decision height (600ft). As pilots completed an approach, the researcher took momentary control of the GCS in order to relinquish control of the current UA and immediately establish control of the next UA.

In four of the six approaches flown per trial, pilots were presented with a scripted conflict. Two of the conflicts were designed to occur prior to ownship reaching final (i.e., "early"), and two were designed to occur once the UA had established itself on final (i.e., "late"). The two remaining approaches flown per trial had no scripted conflict. Nonetheless, it was possible for aircraft that were not scripted to conflict with the UA to interact with the DAA 
system and generate alerts and guidance. The order of presentation of the scripted conflicts varied between scenarios.

Pilots completed a subjective questionnaire and the NASA Task Load Index (NASA-TLX) following each experimental trial. They were also administered a post-simulation questionnaire and debrief at the end of the study.

\section{Measures}

This paper reports on the objective performance of pilots remaining DAA well clear while conducting three different approach procedures in a Class D terminal area. Data sources included output logs from DAIDALUS and VSCS, and video recordings of the TSD. The data allowed for the extraction of three groups of metrics: 1) DAA alerting characteristics, 2) pilot measured response and, 3) loss of DAA well clear.

\section{A. Alert Characteristics}

1. Number of DAA Alerts

A measure of the number of each DAA alert type generated throughout the course of the study. This metric refers to "truth" alerts as any alert logged by the DAA alerting algorithm, regardless of the display configuration. 2. Alert Duration

A measure of the amount of time that an alert remained at the same level before being removed or changing to a new alert level. This metric refers to "truth" alerts as any alert logged by the DAA alerting algorithm, regardless of the display configuration.

\section{B. Measured Response}

Measured response is a set of metrics that were developed to quantify the end-to-end response of task performance by decomposing the task into a discrete set of stages $[14,15]$. Metrics are extracted by measuring the response times between various stages. Table 2 shows the stages of the DAA well clear task as developed by Fern, Rorie, Pack, Shively and Draper [6]. The following three measured response metrics were calculated for the present study.

1. Initial Response Time

A measure of the time it takes a pilot to initiate an input (i.e., edit) into one of the vehicle control interfaces. It is the difference between the first appearance of the alert $\left(\mathrm{T}_{0}\right)$ and the start of the relevant edit $\left(\mathrm{T}_{3}\right)$.

2. Initial Edit Time

A measure of the time it takes a pilot to implement an initial edit into one of the vehicle control interfaces. It is the time between the start of the relevant edit $\left(\mathrm{T}_{3}\right)$ and the first upload to the aircraft $\left(\mathrm{T}_{4 a}\right)$.

3. Aircraft Response Time

A measure of the time it takes the pilot to upload the first maneuver command to the aircraft in response to a traffic alert (i.e., initial response time + initial edit time). It is the time between the initial appearance of the traffic alert $\left(\mathrm{T}_{0}\right)$ and the first upload to the aircraft $(\mathrm{T} 4 \mathrm{a})$.

\section{Loss of DAA Well Clear}

1. Loss of DAA Well Clear Proportion

The proportion of encounters where pilots failed to maintain the DAA well clear separation standard out of all threats predicted to lose DAA well clear, as detected by the DAA system. Only threats that provided the pilot with sufficient time to resolve the conflict (i.e., more than 15 seconds) are included in this metric.

Table 2. Stages of the DAA Well Clear pilot timeline (reprinted with permission from Fern et al. ${ }^{6}$ ).

\begin{tabular}{ll}
\hline Stage & Description \\
\hline $\mathbf{T}_{\mathbf{0}}$ & DAA alert appears on the display \\
$\mathbf{T}_{\mathbf{1}}$ & Pilot notifies ATC of traffic and requests to maneuver around threat \\
$\mathbf{T}_{\mathbf{2}}$ & ATC (nominally) approves pilot's request to maneuver \\
$\mathbf{T}_{\mathbf{3}}$ & Pilot begins interacting with GCS vehicle control interfaces to initiate a maneuver \\
$\mathbf{T}_{\mathbf{4}}$ & Pilot uploads first maneuver to aircraft \\
$\mathbf{T}_{\mathbf{4 b}}$ & Pilot uploads final maneuver to aircraft \\
\hline
\end{tabular}




\section{Loss of DAA Well Clear Categorization}

A diagnosis of losses of DAA well clear, split between pilot-responsible and pilot not-responsible groupings. There were three different categories of pilot-responsible losses of DAA well clear: 1) Pilot Hesitation (pilot had sufficient time to resolve the conflict but failed to upload a resolution to the aircraft in time), 2) Ineffective Maneuver (pilot failed to upload an effective maneuver despite having sufficient time to do so), and 3) Early Return (pilot successfully maneuvered to avoid the initial loss of DAA well clear, but lost separation by returning to course too soon).

There were two major categories of pilot not-responsible losses of DAA well clear: 1) Late Acceleration (the intruder was displayed as a DAA alert with less than 15 seconds prior to the loss of DAA well clear) and 2) Pattern Activity (loss of DAA well clear occurred when the intruder was safely established in the VFR traffic pattern and complying with nominal airport procedures).

3. Loss of DAA Well Clear Severity

The physical extent to which an intruder penetrated the DAA well clear volume. Expressed as a percentage of the DAA well clear threshold that is penetrated, such that a larger percentage equates to greater penetration of the separation threshold. See Phase 1 DAA MOPS Appendix L [2] for details on this metric.

\section{Results}

The measured response metrics were analyzed using a one-way between subjects Analysis of Variance (ANOVA) to compare pilots' responses across the three alerting and guidance display configurations (D1, D2, D3). Descriptive statistics were generated for the alert characteristics, measured response and loss of DAA well clear metrics as a function of alerting and guidance display condition (D1, D2, D3) and encounter location (early, late, unscripted).

\section{A. Alert Characteristics}

1. Number of Alerts

The total number of scripted conflicts that were intended to trigger DAA alerting and result in a loss of Phase 1 DAA well clear in this experiment was 216 (18 pilots by three scenarios per pilot by four scripted encounters per scenario). The number of scripted and unscripted conflicts generating truth DAA alerts (i.e., any alert detected by the DAA system, regardless of the display configuration) is shown in Table 3Table 3. The number (and percentage) of scripted and unscripted conflicts that resulted in a truth DAA alert, by display condition.. While nearly all of the scripted conflicts generated a truth DAA alert (210 of 216), far more truth DAA alerts were generated against unscripted conflicts (326). Scripted encounters, however, were far more likely to progress to a warning truth alert than were unscripted encounters ( $90 \%$ and $46 \%$, respectively).

Table 4 shows the number of truth DAA alerts generated by the three alerting and guidance display configurations. (Note that in D1 corrective and warning alerts were presented as preventive alerts, while in D2 corrective alerts were presented as preventive alerts.) Corrective and warning alerts were the most common alert type for all three configurations. The D3 display configuration resulted in the fewest total number of alerts (331), compared to D1 and D2 (344 and 349, respectively).

Table 3. The number (and percentage) of scripted and unscripted conflicts that resulted in a truth DAA alert, by display condition.

\begin{tabular}{ccccc}
\hline & Number of Intruders & Preventive & Corrective & Warning \\
\hline Scripted & 210 & $147(70 \%)$ & $162(77 \%)$ & $191(90 \%)$ \\
Unscripted & 326 & $160(49 \%)$ & $215(66 \%)$ & $149(46 \%)$ \\
\hline
\end{tabular}

Table 4. The number of preventive, corrective and warning truth alerts generated in each alerting and guidance display configuration.

\begin{tabular}{cccc}
\hline & D1 & D2 & D3 \\
\hline Preventive & 97 & 107 & 103 \\
Corrective & $128^{*}$ & $125^{*}$ & 124 \\
Warning & $119^{*}$ & 117 & 104 \\
TOTAL & 344 & 349 & 331 \\
\hline
\end{tabular}

*The alert was visible to the pilot as a Preventive alert in this alert and guidance display configuration 


\section{Alert Duration}

The mean duration of the 377 corrective truth alerts generated in the experiment was 7.76 seconds (median $=$ 4sec). The distribution of corrective truth alert durations is shown in Fig. 7. The mean duration of the 340 warning truth alerts generated over the course of the study was 23.62 seconds (median $=15 \mathrm{sec}$ ). Of those 340 intruders that generated a warning truth alert, $29 \%$ of them spent 0 seconds as a corrective truth alert, and $64 \%$ of them spent less than 15 seconds as a corrective truth alert.

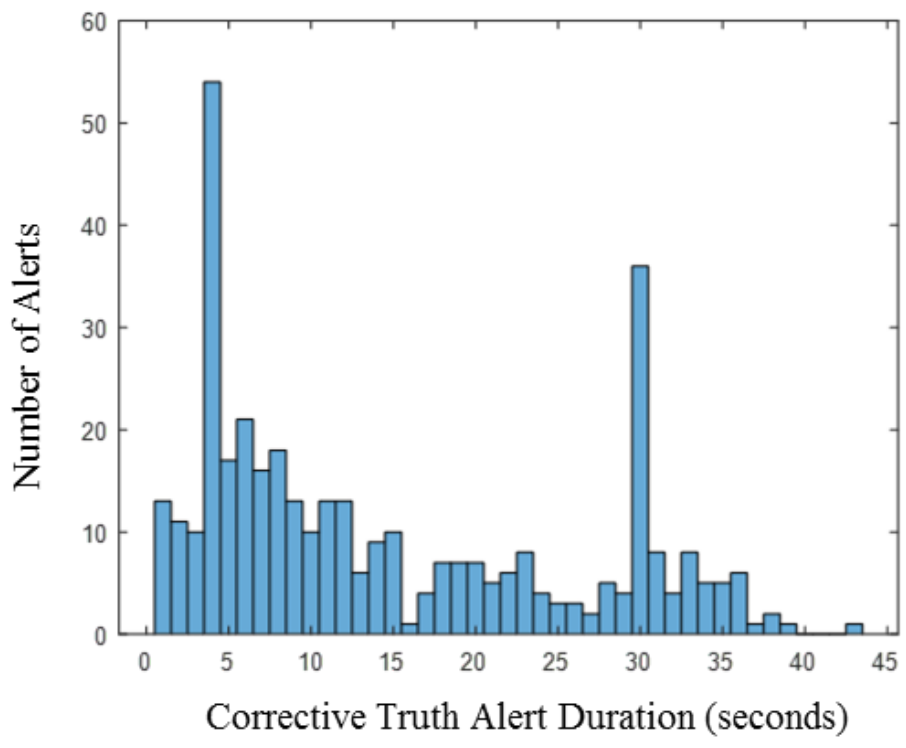

Fig. 7. Distribution of corrective truth alert durations.

\section{B. Measured Response}

1. Initial Response Time

Pilots' initial response times were analyzed separately based on the type of truth alert pilots were responding to, since previous research has established that initial response times tend to be faster to warning-level alerts than corrective-level alerts [16]. No main effect of alerting and guidance display condition was found on initial response times when pilots were responding to corrective alerts, $f(2,15)=3.089, p>.05$. Display condition also failed to have a significant main effect on initial response times to warning alerts, $f(2,15)=2.201, p>.05$. Fig. 8 shows pilots' initial response times as a function of alerting and guidance display configuration and threat type at first alert.

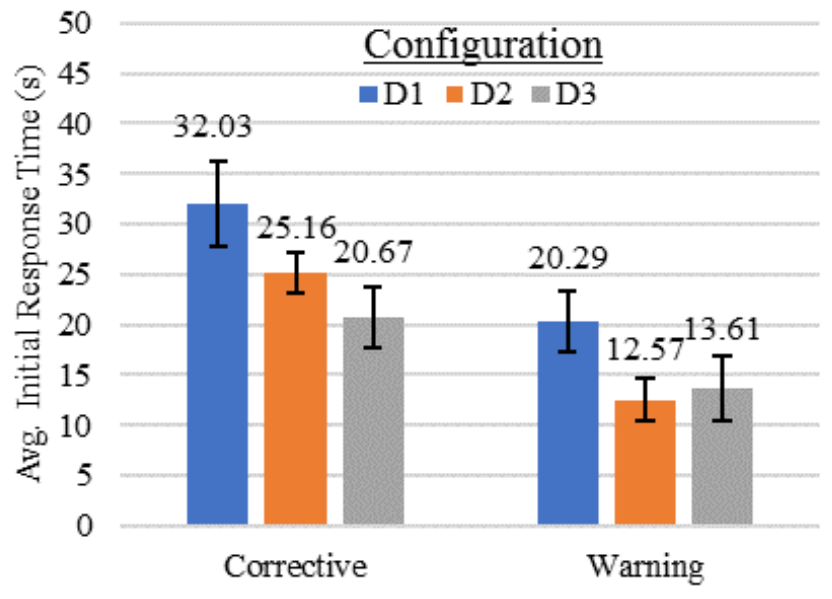

Alert Level (Truth)

Fig. 8 Mean initial response time (and SE) by alerting and guidance display configuration and threat type. 


\section{Initial Edit Time}

No significant main effect of display condition was found on pilot's initial edit times, $f(2,15)=2.658, p>.05$. Initial edit times were slowest in the D1 condition $(M=8.41 \mathrm{sec}, S E=1.22 \mathrm{sec})$, followed by the D3 condition $(M=$ $7.62 \mathrm{sec}, S E=1.27 \mathrm{sec})$, and the D2 condition $(M=5.19 \mathrm{sec}, S E=0.27 \mathrm{sec})$.

3. Aircraft Response Time

As was done with initial response time, pilots' aircraft response times were analyzed separately based on truth alert type. Display configuration did not have a significant main effect on aircraft response times to corrective alerts, $f(2,15)=1.708, p>.05$. The alerting and guidance display configuration also failed to have a significant main effect on aircraft response times to warning alerts, $f(2,15)=2.226, p>.05$. Fig. 9 shows aircraft response times as a function of display configuration and threat type at first truth alert.

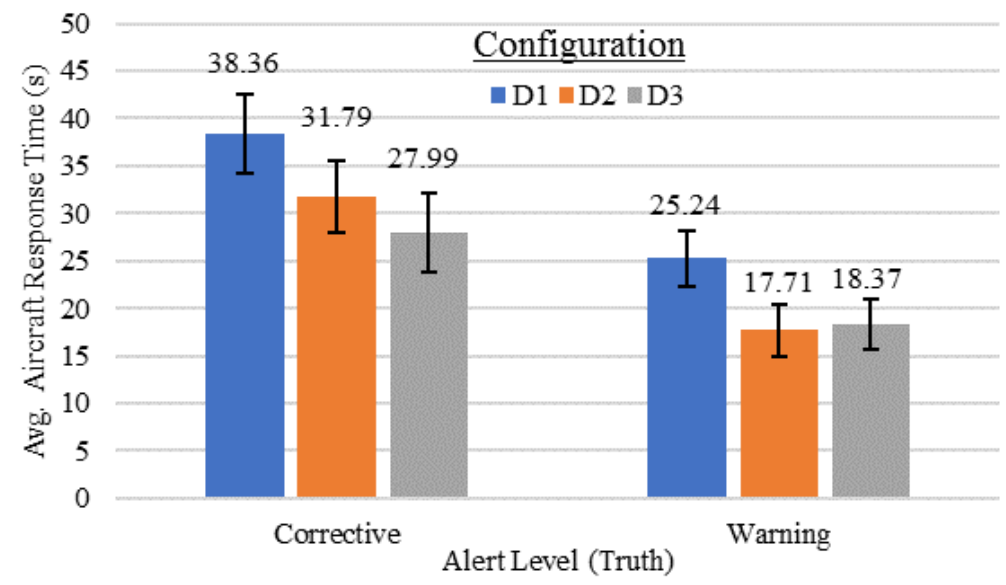

Fig. 9 Mean aircraft response time (and SE) by truth DAA alert level and display configuration.

\section{Loss of DAA Well Clear}

1. Loss of DAA Well Clear Proportion

The proportion of losses of DAA well clear was similar across the display configurations. The D1 alerting and guidance display configuration had the highest proportion of losses of DAA well clear $(M=0.39 ; S E=0.05)$, followed by D2 $(M=0.35 ; S E=0.03)$, and D3 $(M=0.34 ; S E=0.05)$. The proportion of losses of DAA well clear are shown in Fig. 10. The proportion of losses of DAA well clear varied more greatly by encounter location, where late encounters had the highest proportion of losses of DAA well clear $(M=0.82 ; S E=0.05)$, followed by early encounters $(M=0.57 ; S E=0.06)$. Encounters with unscripted intruders had the lowest proportion $(M=0.10 ; S E=$ 0.02). The proportion of losses of DAA well clear by encounter location is shown in Fig. 11.

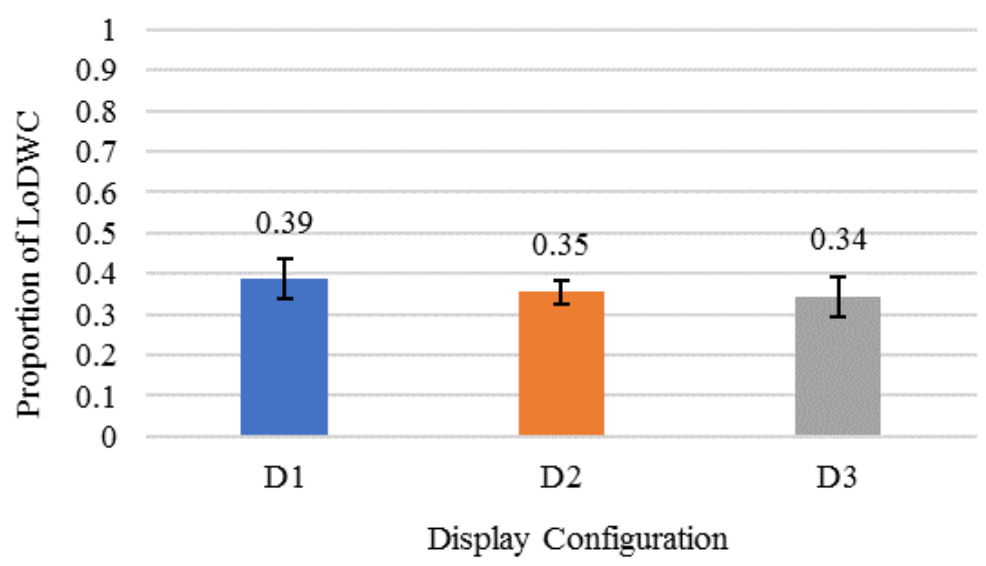

Fig. 10 Proportion of losses of DAA well clear by alerting and guidance display configuration . 


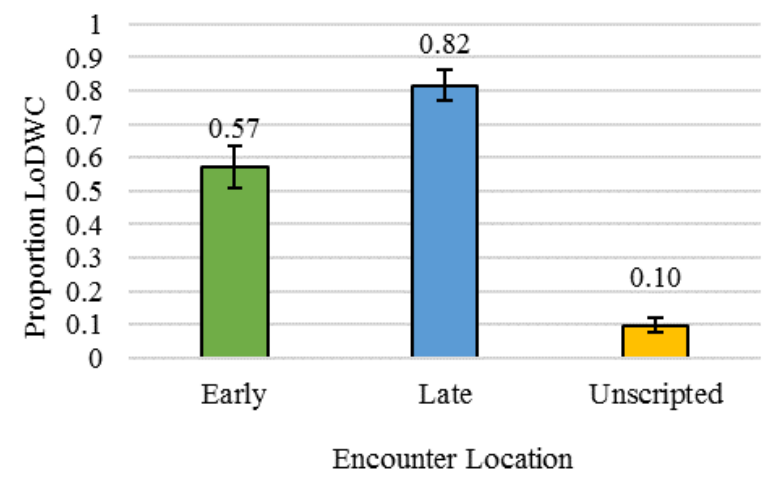

Fig. 11 Proportion of losses of DAA well clear by encounter location.

2. Loss of DAA Well Clear Categorization

Each instance of loss of DAA well clear was categorized according to its cause. Table 5 shows the proportion of loss of DAA well clear type by pilot responsibility and alerting and guidance display configuration. The most common pilot-responsible cause of loss of DAA well clear was pilot hesitation and was especially pronounced in the D1 configuration. Ineffective maneuvers and early returns to course were less common, but more consistent across configurations. The most common cause of a loss of DAA well clear where the pilot was not responsible was a late acceleration by the intruding aircraft.

Table 5. Loss of DAA Well Clear Categorization by pilot responsibility.

\begin{tabular}{llccc}
\hline & \multicolumn{1}{c}{ LoDWC Category } & D1 & D2 & D3 \\
\hline \multirow{2}{*}{ Pilot Responsible } & Pilot Hesitation & $48 \%$ & $26 \%$ & $33 \%$ \\
& Ineffective Maneuver & $14 \%$ & $16 \%$ & $20 \%$ \\
& Early Return & $6 \%$ & $11 \%$ & $11 \%$ \\
\hline \multirow{2}{*}{ Pilot Not Responsible } & Late Acceleration & $29 \%$ & $39 \%$ & $31 \%$ \\
& Pattern Activity & $3 \%$ & $9 \%$ & $4 \%$ \\
\hline
\end{tabular}

3. Loss of DAA Well Clear Severity

The severity of losses of DAA well clear (SLoWC) that occurred are shown in Fig. 12. Severity ranged from $20.69 \%$ (D2) to $28.43 \%$ (D1) penetration of the DAA well clear threshold across the alerting and guidance display configurations. The loss of DAA well clear severity varied more greatly by encounter location, where late encounters had the highest SLoWC $(M=29.91 ; S E=3.26)$, followed by early encounters $(M=21.48 ; S E=3.96)$. The encounters with unscripted intruders had the lowest severity $(M=7.90 ; S E=2.29)$. The severity of losses of DAA well clear by encounter location is shown in Fig. 13.

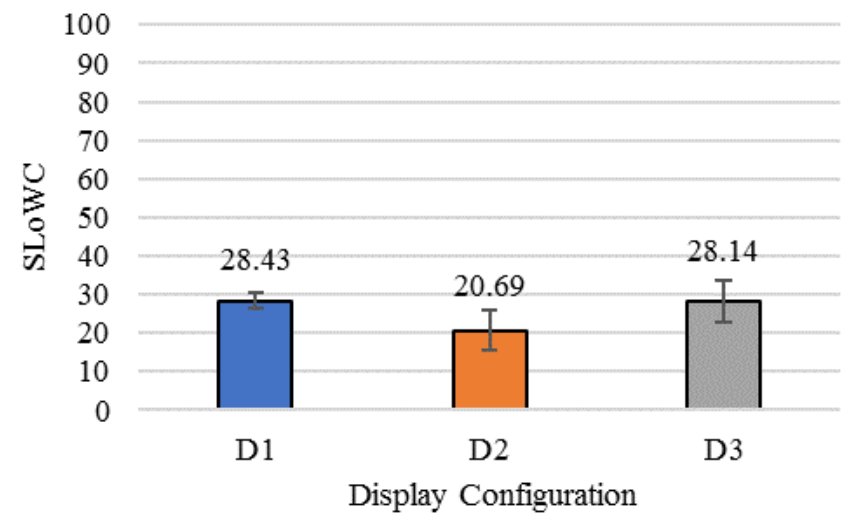

Fig. 12 Mean severity of loss of DAA well clear (SLoWC; and SE) by alerting and guidance display configuration. 


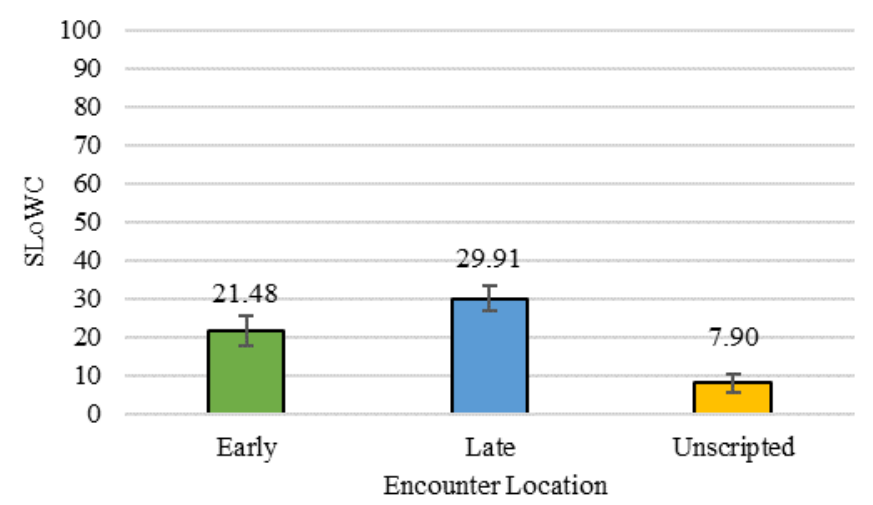

Fig. 13 Mean severity of loss of DAA well clear (SLoWC; and SE) by encounter location.

Fig. 14 and Table 6 show the distribution and number of high-severity of losses of DAA well clear (i.e., SLoWC $>50 \%$ ). The D3 alerting and guidance display configuration had the largest percentage of losses of DAA well clear with severity over 50 (20\%) and 70 (7\%) compared to D1 and D2. Similarly, late encounters had the largest percentage of severity over $50(20 \%)$ and $70(7 \%)$ compared to early and unscripted encounters.
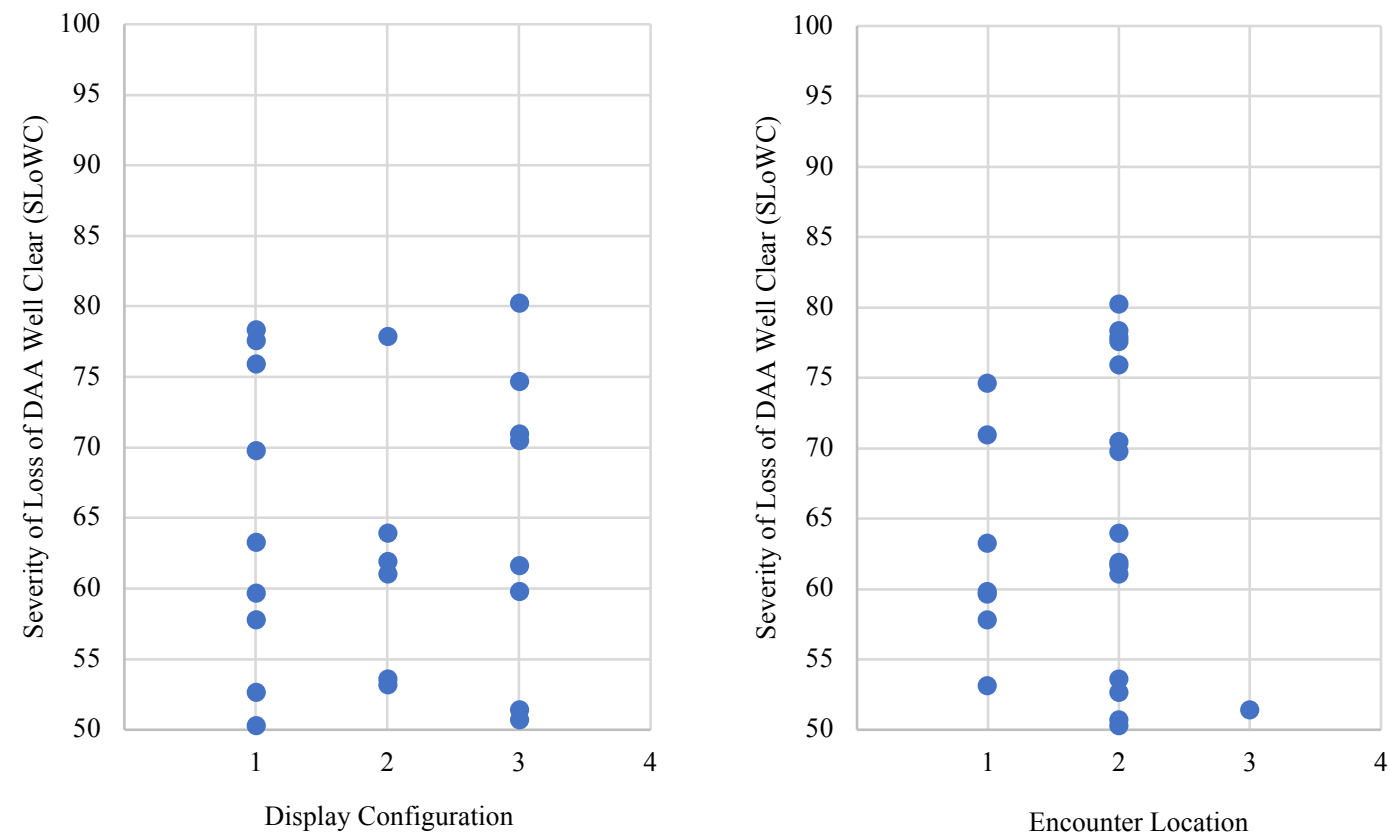

Fig. 14 Distribution of losses of DAA well clear with a severity (SLoWC) of 50 or greater by display configuration (left) and encounter location (right).

Table 6. Number (and percentage) of severity of losses of DAA well clear (SLoWC) greater than 50 and 70 by display configuration and encounter location.

\begin{tabular}{cccc}
\hline & D1 & D2 & D3 \\
\hline SLoWC Above 50 & $10(15 \%)$ & $7(12 \%)$ & $11(20 \%)$ \\
SLoWC Above 70 & $3(5 \%)$ & $1(2 \%)$ & $4(7 \%)$ \\
\hline & Early & Late & Unscripted \\
\hline SLoWC Above 50 & $9(16 \%)$ & $18(20 \%)$ & $1(3 \%)$ \\
SLoWC Above 70 & $2(3 \%)$ & $6(7 \%)$ & 0 \\
\hline
\end{tabular}




\section{Discussion}

The above results indicate that the three different alerting and guidance display configurations evaluated in this experiment had very little effect on pilot responses to the Phase 1 DAA alerting and guidance. There were no significant effects of display configuration on any of the three measured response metrics for pilots responding to corrective or warning alerts. In addition, the proportion of LoDWC was nearly identical across the three displays $(39 \%, 35 \%$ and $34 \%$ respectively for D1, D2, and D3), with the majority of pilot responsible LoDWC being the result of pilots responding too slowly to DAA alerts (Table 5). That said, there was a small benefit seen for D2 in the severity of LoDWC metrics, where D2 had the lowest average severity overall in addition to having the lowest percentage of high severity LoDWC compared to the other two display conditions. Thus, pilots performed similarly at the task of minimizing LoDWC severity when there was essentially no DAA alerting and guidance (D1) compared to when the full Phase 1 alerting and guidance was present (D3).

The lack of difference in performance between the three alerting and guidance display conditions may have been due to the high number of alerts throughout the experimental trials. While 210 of the 216 scripted encounters resulted in truth alerts, 326 unscripted encounters also generated alerts. The majority of these unscripted encounters were generated by aircraft in the VFR traffic pattern that were considered safely separated given local procedures for the Class D terminal area. Although a large number of unscripted encounters generated DAA truth alerts and guidance, only $10 \%$ of those encounters resulted in a LoDWC (Fig. 11); early and late scripted encounters were much more likely to results in LoDWCs (57\% and 82\%, respectively). When LoDWCs did occur, the unscripted encounters were much less severe, penetrating the DAA well clear threshold by $8 \%$ on average, with a single LoDWC considered high severity (Table 6). In comparison, early and late encounters resulted in higher average severity ( $21 \%$ and $30 \%$ penetration of the DAA well clear threshold, respectively) and had higher numbers of high severity LoDWC (11 and 24, respectively). Thus, encounter location (early, late, unscripted) had a much larger impact on pilot performance than did the alerting and guidance display configuration.

Another notable finding from the current study was the limited utility of corrective truth alerts. According to the prescribed alerting parameters used in this study (Table 1), a corrective alert should be issued 55 seconds prior to a loss of DAA well clear, with the transition to a warning occurring at 25 seconds prior to a loss of DAA well clear. Warning truth alerts had an average duration $(24 \mathrm{sec})$ consistent with these alerting parameters, but the corrective truth alerts did not. Corrective truth alerts should be visible for 30 seconds prior to the threat elevating to a warninglevel alert. This time is intended to provide pilots with sufficient time to contact ATC to coordinate a response, which, according to previous research, takes approximately 10 to 15 seconds [17]. As reported above, corrective truth alerts in the current study lasted, on average, 8 seconds, severely undermining the purpose of the corrective alert in the Phase 1 DAA system by providing insufficient time for pilots to carry out the ATC coordination. The cause of the drop in corrective truth alert durations is most likely the dynamic and closely-spaced nature of the terminal environment, where traffic can progress quickly from a non-alert, to an imminent loss of DAA well clear.

The pilot response times observed in the current study were considerably slower than has been observed in previous research with the Phase 1 DAA system in the en route environment. Rorie et al. [10] found aircraft response times to corrective and warning alerts to average 18 and 10 seconds, respectively. Aircraft response times to corrective and warning alerts in the current experiment averaged 33 and 20 seconds, respectively, at least 10 seconds slower than those observed for Rorie et al. The slower response times overall is likely the result of both the excessive alerting of the DAA system, as well as the fact that the corrective and/or warning alerts were not visible to pilots in the D1 and D2 display conditions. The slower response times overall in this experiment is consistent with the observation that the most common cause of LoDWC was pilot hesitation, an indication that pilots were not immediately responding to alerts that were generated by the DAA system. The lack of responsiveness resulted not just in a higher proportion of LoDWC than has been seen in prior simulation, but also more severe instances of LoDWC. The proportion (1-3\%) and severity (5-9\% penetration) of LoDWC reported in Rorie et al. is substantially lower than was seen in the current study, without a single instance of LoDWC severity above $30 \%$. For reference, there were 17 instances pilot-responsible losses of DAA well clear with a severity greater than $50 \%$ in the present experiment.

Taken together, these results indicate that due to the high numbers of alerts across all scenarios and display configurations, a result of the closer proximity of aircraft in a terminal area, the Phase 1 DAA system was providing limited useful information to pilots about when an avoidance maneuver was actually necessary. This resulted in pilots taking longer to respond and having more frequent and more severe losses of DAA well clear compared to operations with the Phase 1 DAA system in en route environments where aircraft are further apart. The most likely reason for this is that the Phase 1 DAA well clear definition was too big to distinguish between safely separated aircraft and legitimate threats. The fact that pilots with essentially no DAA alerting and guidance (D1) performed 
roughly the same as pilots with the full Phase 1 alerting and guidance structure (D3) supports the hypothesis that pilots were left to guess as to when a maneuver was really necessary.

\section{Conclusion}

The present study provides empirical evidence that the Phase 1 DAA well clear definition, alerting and guidance is insufficient to support safe UAS terminal area operations. A new DAA well clear definition, as well as updated alerting and guidance requirements, should be designed to accommodate the typical operations performed in the terminal environment. The DAA well clear definition and alerting parameters, for instance, should refrain from alerting against traffic in the VFR traffic pattern, whether that traffic is in the downwind or turning in front of the UAS. By not alerting against such traffic, the DAA system will better support pilots' detection of legitimate threats. The alerting and guidance structure should also be investigated further, particularly the use of the corrective alert. The dynamics of the terminal environment may not provide sufficient time to realize the benefits of the corrective alert, namely the explicit coordination with ATC. Finally, how and when the new terminal-area DAA well clear criteria is applied to surrounding traffic should be studied relative to its impact on pilot performance. Any changes made to the size of the DAA well clear definition or to the DAA alerting and guidance structure should not negatively impact pilots' interaction with the DAA system.

\section{References}

[1] RTCA. "DO-362 - Command and control (C2) data link minimum operational performance standard (MOPS) (Terrestrial)," RTCA Inc., Washington, D.C., 2016.

[2] RTCA. "DO-365 - Minimum operational performance standards (MOPS) for detect and avoid (DAA) systems," RTCA Inc., Washington, D.C., 2017.

[3] RTCA. "DO-366 - Minimum operational performance standards (MOPS) for air-to-air radar for traffic surveillance," RTCA Inc., Washington, D.C., 2017.

[4] Title 14 Code of Federal Regulations (14CFR) Part 91, sections .3, .111, .113(b), and .181(b).

[5] Cook, S. P., Brooks, D., Cole, R., Hackenberg, D., and Raska, V. "Defining well clear for unmanned aircraft systems, ” AIAA Infotech@Aerospace, 1-20, 2015.

[6] Fern, L., Rorie, R.C., Pack, J.S., Shively, R.J. \& Draper, M.H. "An evaluation of Detect and Avoid (DAA) displays for unmanned aircraft systems: The effect of information level and display location on pilot performance." Proceedings of 15th AIAA Aviation Technology, Integration, and Operations Conference, 2015.

[7] Rorie, R. C. and Fern, L. "The impact of integrated maneuver guidance information on UAS pilots performing the Detect and Avoid task." In Proceedings of the 59th Human Factors and Ergonomics Society Annual Meeting, 2015.

[8] Rorie, R.C., Fern, L., and Shively, R.J. "The impact of suggestive maneuver guidance on UAS pilots performing the detect and avoid function." AIAA Infotech@,Aerospace, 2016.

[9] Rorie, R.C., and Fern, L.F., "An interoperability concept for detect and avoid and collision avoidance systems: results from a human-in-the-loop simulation," $18^{\text {th }}$ AIAA Aviation Technology, Integration, and Operations Conference, Atlanta, GA, 2529 June 2018.

[10] Rorie, R.C., Fern, L., Roberts, Z., Monk, K., Santiago, C., and Shively, R.J. "Validation of minimum display requirements for a UAS detect and avoid system" AIAA Infotech@, Aerospace, 2017.

[11] RTCA. "Terms of reference RTCA special committee 228 minimum performance standards for unmanned aircraft systems Rev 3,” paper No. 239-16/PMC-1527. RTCA Inc., Washington, DC, 2013.

[12] Feitshans, Gregory L., Allen J. Rowe, Jason E. Davis, Michael Holland, and Lee Berger. "Vigilant spirit control station (VSCS)_'The face of COUNTER'." In Proceedings of AIAA Guidance, Navigation and Control Conf. Exhibition, 2008.

[13] Muñoz, C., Narkawicz, A., Hagen, G., Upchurch, J., Dutle, A., Consiglio, M., and Chamberlain, J., "DAIDALUS: Detect and Avoid Alerting Logic for Unmanned Systems," 34th Digital Avionics Systems Conference (DASC), IEEE/AIAA, 2015, pp. 5A1-1.

[14] Shively, R.J., Vu, K-PL., and Buker, T.J. "Unmanned Aircraft System Response to Air Traffic Control Clearances Measured Response." Proceedings of the Human Factors and Ergonomics Society Annual Meeting. Vol. 57. No. 1. SAGE Publications, 2013.

[15] Vu, K-PL, Morales, G., Chiappe, D., Strybel, T.Z., Battiste, V., Shively, R.J., and Buker, T.J. "Influence of UAS pilot communication and execution delay on controller's acceptability ratings of UAS-ATC interactions." Digital Avionics Systems Conference (DASC), 2013 IEEE/AIAA 32nd, pp. 6D4-1, 2013.

[16] Monk, K.J., Roberts, Z., Fern, L., \& Rorie, C. "Utility of visual and auditory warning alerting for traffic avoidance." Proceedings of the Human Factors and Ergonomics Society 62nd Annual Meeting, in press, 2018.

[17] Fern, L. and Rorie, R.C.,. "An Examination of Unmanned Aircraft Systems Pilots' Interaction with Air Traffic Control while Responding to Detect and Avoid Conflicts," Air Traffic Control Quarterly, 23(2-3), pp.113-135, 2015. 